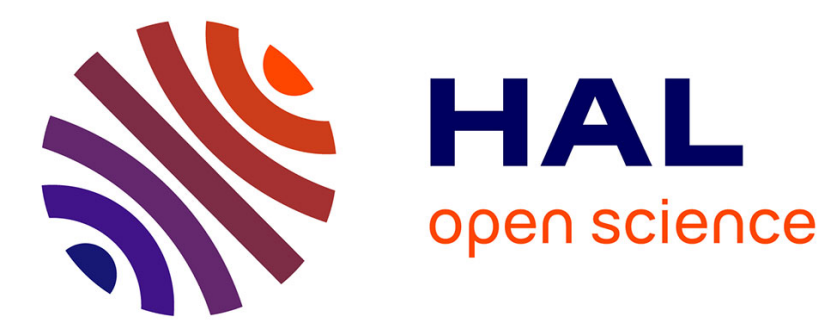

\title{
Relaxation of the single-mode emission conditions in extended-cavity semiconductor lasers with a self-organizing photorefractive filter
}

\author{
Antoine Godard, Gilles Pauliat, Gérald Roosen, Eric Ducloux
}

\section{To cite this version:}

Antoine Godard, Gilles Pauliat, Gérald Roosen, Eric Ducloux. Relaxation of the single-mode emission conditions in extended-cavity semiconductor lasers with a self-organizing photorefractive filter. Applied optics, 2004, 43 (17), pp.3543-3547. hal-00866991

HAL Id: hal-00866991

https://hal-iogs.archives-ouvertes.fr/hal-00866991

Submitted on 27 Sep 2013

HAL is a multi-disciplinary open access archive for the deposit and dissemination of scientific research documents, whether they are published or not. The documents may come from teaching and research institutions in France or abroad, or from public or private research centers.
L'archive ouverte pluridisciplinaire HAL, est destinée au dépôt et à la diffusion de documents scientifiques de niveau recherche, publiés ou non, émanant des établissements d'enseignement et de recherche français ou étrangers, des laboratoires publics ou privés. 


\title{
Relaxation of the single-mode emission conditions in extended-cavity semiconductor lasers with a self-organizing photorefractive filter
}

\author{
Antoine Godard, Gilles Pauliat, Gérald Roosen, and Éric Ducloux
}

\begin{abstract}
Commercial $1.55-\mu \mathrm{m}$ extended-cavity semiconductor lasers provide single-mode operation that can be continuously tuned over a range larger than $100 \mathrm{~nm}$ without mode hopping. But such performance requires delicate factory adjustment and high mechanical stability of the external cavity. Furthermore, at high emission power the tuning range is limited to small values because of the annoying multimode operations that sometimes occur. We have shown that the alignment constraints can be relaxed by use of an intracavity photorefractive filter. Here we present new results obtained with a crystal with low absorption and high photorefractive gain. We demonstrate that, without inducing excessive additional loss, we can preserve single-mode emission at an output power higher than the maximum power obtained in the absence of a photorefractive crystal over the full tuning range of the laser. (C) 2004 Optical Society of America
\end{abstract}

OCIS codes: $140.5960,160.5320,190.7070,140.3570,050.7330$.

\section{Introduction}

In the testing of optical communication networks and components, 1.55- $\mu \mathrm{m}$-wavelength-tunable singlemode extended-cavity semiconductor lasers (ECLs) are widely used. An ECL is a semiconductor amplifier strongly coupled to an external cavity through its antireflection-coated facet. ${ }^{1-8}$ To produce tunable single-mode emission, the external cavity contains a selective element that is usually a grating. Minimum-loss wavelength $\lambda_{G}$ corresponds to the wavelength that is retroreflected by the external cavity and thus is optimally backcoupled into the semiconductor amplifier. Its value is fixed by the selective element angle. For other wavelengths the return beam shifts from the best coupling position. Consequently, the loss increases with the detuning.

A. Godard, G. Pauliat (gilles.pauliat@iota.u-psud.fr), and G. Roosen are with the Laboratoire Charles Fabry de l'Institut d'Optique, Centre National de la Recherche Scientifique, Université Paris-Sud, Centre Scientifique d’Orsay, Bâtiment 503, F-91403 Orsay Cedex, France. E. Ducloux and A. Godard are with the Photonics Division, NetTest 45, Avenue Jean Jaurès, F-78344 Les Clayes sous Bois, France.

Received 11 July 2003; revised manuscript received 17 February 2004; accepted 30 March 2004.

0003-6935/04/173543-05\$15.00/0

(C) 2004 Optical Society of America
Oscillating-mode wavelength $\lambda_{0}$ is thus selected by external-cavity selectivity to be close to $\lambda_{G}$.

Current commercial devices can be continuously tuned over a range larger than $100 \mathrm{~nm}$, but this wide tunability requires delicate factory adjustment and high stability of the external cavity. Indeed, the exact value of $\lambda_{0}$ depends on the cavity length, and mismatch $\delta \lambda=\lambda_{0}-\lambda_{G}$ between the lasing wavelength and the wavelength of minimum loss must be kept inside a restricted range to prevent mode hopping or multimode operation that could occur at high power near the boundaries of the accessible range of $\delta \lambda . .^{5-8}$ The cavity length and the frequency selective element must thus be accurately and synchronously adapted to each other.

In Refs. 9 and 10 it was shown that the insertion of a photorefractive crystal inside the extended cavity increases the accessible range of $\delta \lambda$, thus relaxing the fabrication tolerances. These photorefractive crystals are dynamic holographic media in which a hologram spontaneously develops as a refractive-index replica of the illumination pattern. ${ }^{11}$ Inside the cavity this illumination pattern originates from the oscillating-mode standing-wave interference. This pattern thus records a Bragg grating inside the crystal that acts as a frequency filter and, in this way, modifies the loss for each mode. Inasmuch as it is written by the oscillating mode, this hologram creates an adaptive spectral filter that decreases the loss for 


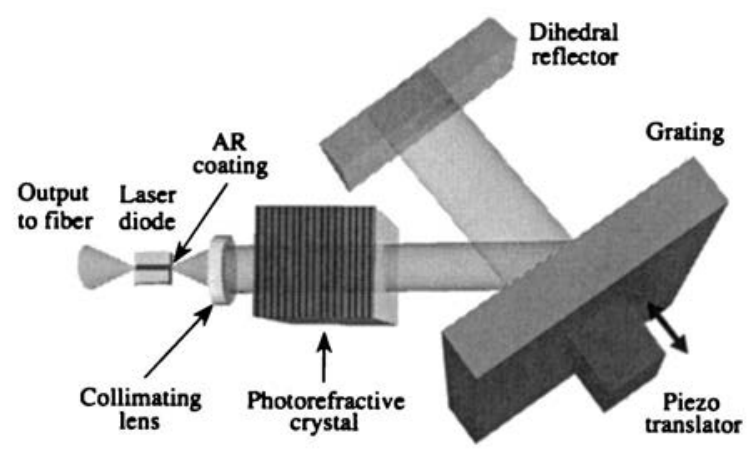

(a)

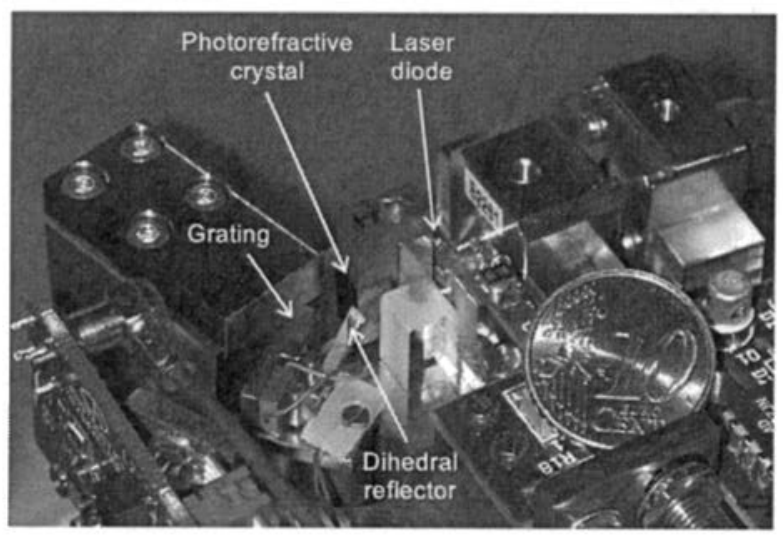

(b)

Fig. 1. (a) Sketch and (b) photograph of the grating-tuned extended-cavity laser, which comprises an antireflection- (AR-) coated laser, a collimating optical system, a photorefractive crystal, and a Littman-mounted grating.

this lasing mode and thus enhances modal selectivity. ${ }^{9,10,12-14}$

Here we demonstrate that, in addition to extending the accessible range of $\delta \lambda$ in the full tuning range, the photorefractive crystal also efficiently prevents multimode operation. In this way, without incurring excessive additional loss, single-mode operation is maintained at output power levels that are higher than the maximum power obtained in the absence of a photorefractive crystal.

\section{Characteristics of the Laser Cavity and the Photorefractive Crystal}

The optical configuration of the laser, shown in Fig. 1, has been specially adapted to facilitate insertion and removal of the photorefractive crystal. Most of the other components are glued in place guarantee excellent stability.

The ECL consists of a multiple-quantum-well InGaAsP laser diode optically coupled to an external cavity through its antireflection-coated facet (residual reflectivity $R_{\mathrm{AR}}<10^{-3}$ ). The opposite, uncoated, facet serves as the output-coupling mirror. The external cavity consists of a collimating lens and

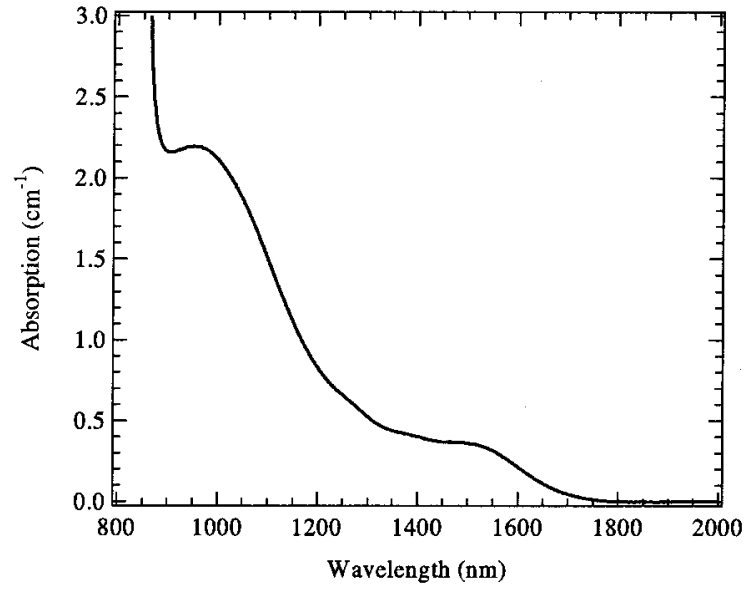

Fig. 2. Absorption spectrum of the CdTe sample.

a Littman-mounted grating as a wavelengthselective device. ${ }^{7}$ The photorefractive crystal is inserted between the collimating lens and the grating, as shown by Fig. 1.9,10 We have chosen a CdTe crystal for its high photorefractive sensitivity near 1.55 $\mu \mathrm{m} .{ }^{15}$

This sample, which is $4.5 \mathrm{~mm}$ thick, was grown at Imarad Imaging Systems Ltd., Israel. It is cut with [001] polished faces. The beam-propagates along the (001) direction, whereas the other faces are [110] and $[1 \overline{1} 0]$. The polarization of the lasing wave is along the (110) direction.

In previous research reported in Refs. 9 and 10, the absorption of the former sample was too high and thus dramatically reduced the performance of the laser. These losses originated from the optical absorption but also from microscopic defects such as inclusions. As shown in Fig. 2, the losses of our new crystal vanish above $1800 \mathrm{~nm}$. This result indicates that the inclusions are absent from this new sample and that these losses correspond mostly to the active absorption that is necessary to produce the photorefractive effect. This absorption is roughly flat and inferior to $0.5 \mathrm{~cm}^{-1}$ in the full tuning range of the laser.

In the expectation of its use for intracavity filtering in the tunable ECL, we also measured the counterpropagating two-wave-mixing gain of the crystal as a function of the wavelength in the full tuning range of the laser. As shown by Fig. 3, the photorefractive gain changes only slightly over the measured range, which is larger than $100 \mathrm{~nm}$. This is consistent with the absorption spectrum presented in Fig. 2, whose active absorption near $1.55 \mu \mathrm{m}$ has a bandwidth larger than the ECL's tuning range.

One should note that the measured photorefractive gain is higher than the usual reported values for vanadium-doped CdTe at $1.55 \mu \mathrm{m} .{ }^{15}$ We attribute this, high gain to a lower hole-electron competition at $1.55 \mu \mathrm{m}$ in this new sample. This assumption has been confirmed by our gain measurements. Similarly to measurements of previous samples, the photorefractive gain changes its sign between 


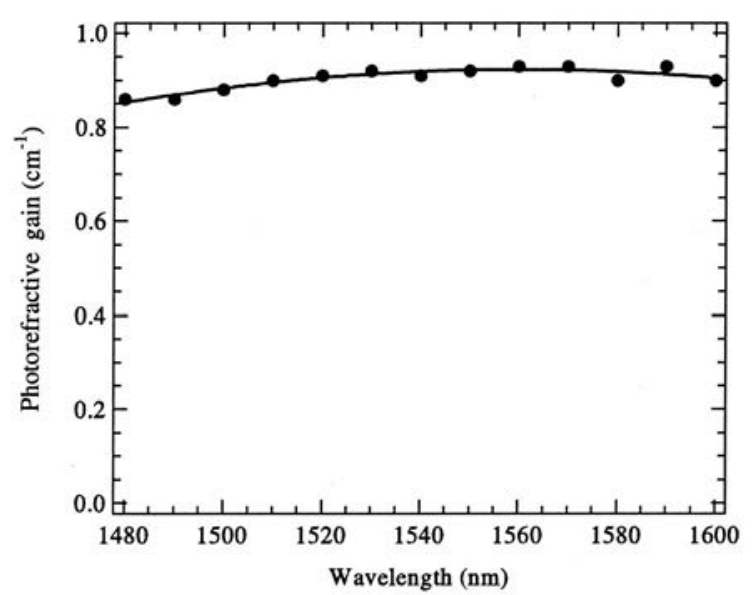

Fig. 3. Counterpropagating two-wave mixing photorefractive gain of the CdTe sample as a function of wavelength. The solid curve is a guide for the eye, not a theoretical fit.

wavelengths of $1.55 \mu \mathrm{m}$ and $1064 \mathrm{~nm}$ : At $1064 \mathrm{~nm}$ the photorefractive effect is due mainly to electron transport, whereas the hole contribution is the largest at $1550 \mathrm{~nm}$. Nevertheless, unlike for previous samples, for the new sample the photorefractive gain is higher at $1550 \mathrm{~nm}$ than at $1064 \mathrm{~nm}$, indicating that the hole-electron competition is now larger at 1064 than at $1550 \mathrm{~nm}$.

Therefore, with its high gain and low absorption, the sample seems to be suited to implement a selforganizing filter in the ECL. For this purpose the two polished faces are antireflection (AR) coated from 1480 to $1600 \mathrm{~nm}$, and the normal of these faces is $2^{\circ}$ tilted relative to the beam axis to prevent any parasitic feedback in the laser diode. The threshold value of the ECL at $1.55 \mu \mathrm{m}$ increases only slightly, from 23 to 27 $\mathrm{mA}$, with the crystal, as one can see for the typical light-current characteristics plotted in Fig. 4. The ripple in the characteristics is due to the residual reflectivity of the diode facet's AR coating that induces a parasitic Fabry-Perot effect (see Section 4 below for a more-detailed discussion of this issue).

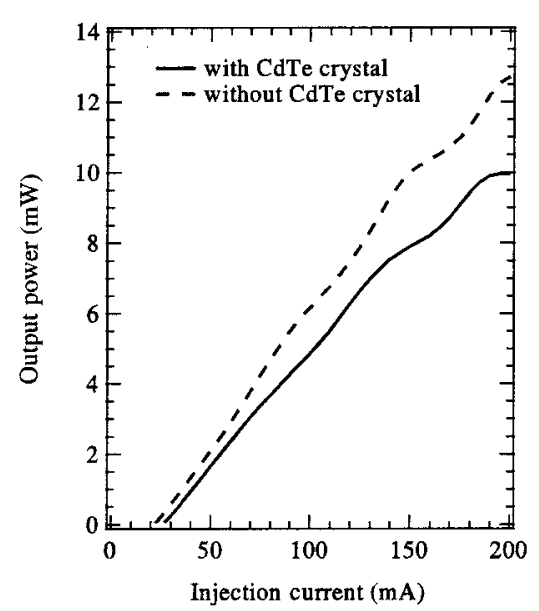

Fig. 4. Typical characteristics of light versus current at $1.55 \mu \mathrm{m}$.

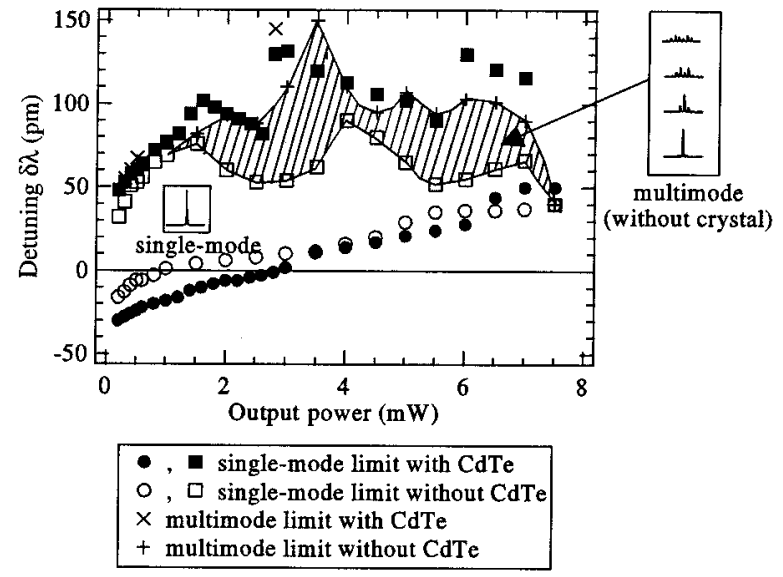

Fig. 5. Accessible range of $\delta \lambda$ under stable single-mode operation as a function of optical power at $1.6 \mu \mathrm{m}$ with and without a CdTe crystal. The data denoted "single-mode limit" correspond to mode hops toward another single-mode state or to the appearance of multimode operation; the data denoted "multimode limit" correspond to mode hops from a multimode state toward a single-mode state.

In what follows, we describe the improvement of the single-mode operating range of the ECL by use of the photorefractive filter. We measure the expansion of the accessible range of the detuning as a function of the optical power when the crystal is inserted inside the cavity, especially when multimode operation occurs without a crystal.

\section{Measurements of the Stable Single-Mode Operation Range}

By using a piezo ceramic (Fig. 1), we can change the cavity length without modifying the grating angle. Doing so will allow mismatch $\delta \lambda$ between the wavelength of lasing mode $\lambda_{0}$ and minimum-loss wavelength $\lambda_{G}$ to be scanned. We measured the value of $\delta \lambda$ by use of a wavemeter with a resolution of \pm 1 part in $10^{6}$. Concurrently, we used a scanning FabryPerot interferometer to check that the output spectrum was actually single mode.

The accessible range $\delta \lambda$ for stable single-mode operation, whose measurements at $1.6 \mu \mathrm{m}$ are plotted in Fig. 5, was measured according to the following procedure, which was the same with and without a CdTe crystal inside the cavity. Near threshold and at $\delta \lambda=0$ the laser oscillated on mode 0 . We increased the wavelength of mode 0 by lengthening the cavity, using the piezo translator, until a mode hop occurred toward shorter wavelengths for a given value of $\delta \lambda$ (these values are represented by squares in Fig. 5). Then the cavity was shortened to recover mode 0 . This shortening was continued until mode hopping occurred toward the longer wavelengths, represented by circles in Fig. 5. Next, the cavity was again lengthened to return to mode 0. Afterward, the same procedure was carried out for each increasing value of the optical power.

For output power superior to $2 \mathrm{~mW}$ without a crystal and in specific situations with a crystal, the upper 
boundaries of the single-mode domain delimited by squares in Fig. 5 correspond not to mode hops but to the emergence of multimode operations continuously from the single-mode states. The actual mode hops happen from the multimode states at the points denoted by crosses.

As shown in Fig. 5, the photorefractive filter always increases the accessible range of $\delta \lambda$, especially at high power when a large multimode operations domain appears without a crystal. The stable range always shifts toward positive values of $\delta \lambda$ when the output power increases. Furthermore, in the case of Fig. 5 it broadens until the power reaches the value of 2 $\mathrm{mW}$. Then, for higher power, in the without-crystal configuration the multimode operation domain appears and the stable range narrows and eventually closes up.

The photorefractive filter efficiently suppresses most multimode operations. Moreover, the stable range remains wide open, even at high optical-power levels, as shown in Fig. 5. Here we present only the measurements at $1.6 \mu \mathrm{m}$, but this kind of improvement of the stable single-mode range is efficient in the full tuning range of the ECL, from 1480 to 1600 nm. Moreover, we did not note any degradation of long-term stability owing to insertion of the crystal.

\section{Discussion}

Because the intermode spacing is much smaller than the semiconductor amplifier gain spectrum's bandwidth, the gain roll-off can be neglected. The stable single-mode operation range boundaries should thus correspond to the values of $\delta \lambda$ at which the loss of one of the side modes equals the lasing-mode loss and should not depend on the optical power; in fact, as we can see from Fig. 5, this loss strongly depends on the optical power. The power dependence of the accessible range of $\delta \lambda$ is due to the mode-coupling phenomena and to the nonzero reflectivity of the semiconductor chip's antireflection-coated facet.

The mode coupling is a result of nonlinear phenomena inside the active medium that induce wave mixing. ${ }^{8,16-18}$ The beating of the main mode with nonlasing side modes leads to modulations of various parameters of the medium. A comprehensive analysis of the wave-mixing processes is beyond the scope of this paper. Nevertheless we can mention that they produce a nonsymmetrical mode coupling that is different for the shorter-wavelength side modes and the longer wavelength side modes and fully explain the wavelength shift of the stable single-mode operation range.

Moreover, we can also note, from Fig. 5, a modulation of the boundaries of the accessible range of $\delta \lambda$ as a function of the optical power. This modulation is due to the Fabry-Perot effect between the two facets of the semiconductor chip whose phase condition changes with the optical power. In this way, the differential loss is altered when power increases, and consequently the mode-hopping condition is changed. The modulation that is due to the Fabry-Perot effect is much smaller for circles than for squares because

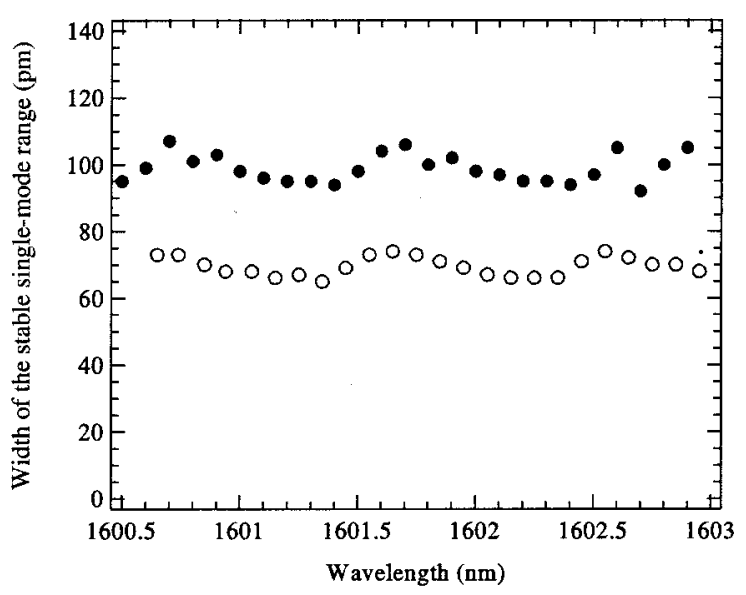

(a)

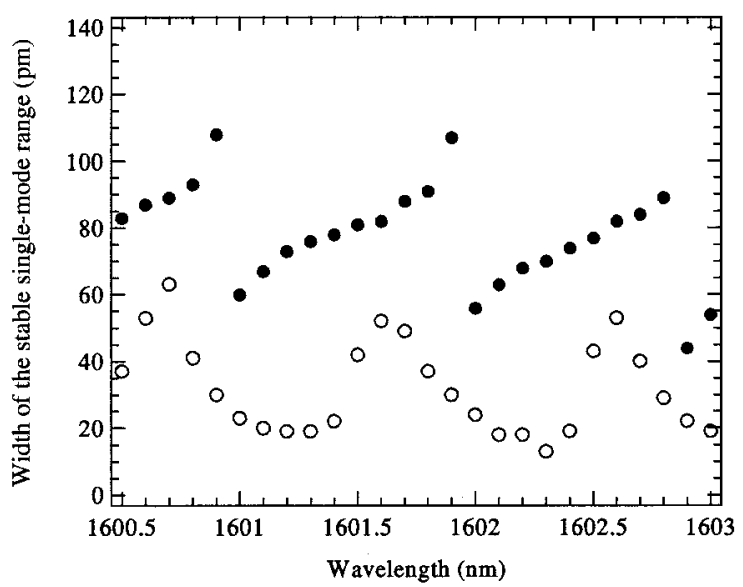

(b)

Fig. 6. Width of the stable single-mode range as a function of wavelength at constant output powers of (a) 1 and (b) $5 \mathrm{~mW}$. Filled circles and open circles, measurements with a CdTe crystal inside the cavity and without a crystal, respectively.

the magnitude of detuning $\delta \lambda$ is larger for squares than for circles. The relative value of the residual reflectivity of the AR coating with respect to the external cavity reflectivity is thus larger; consequently the contrast of the Fabry-Perot effect is also larger.

To investigate in more detail the effect of the residual reflectivity of the AR coating we measured the width of the stable single-mode range as a function of the wavelength that is selected by the diffraction grating when the output power is kept constant. Figure 6 shows such measurements for 1- and 5-mW output power near $1.6 \mu \mathrm{m}$. We can observe a periodic ripple of the width of the stable single-mode range. The value of this period is $\sim 1 \mathrm{~nm}$, which corresponds to a 1.28-mm-thick Fabry-Perot effect, which is consistent with the optical length of the laser chip. It is noticeable that, for a given position in the ripple period, the width of the stable single-mode range is always increased when the crystal is inserted inside the laser cavity.

The amplitude of the ripple is larger when the 
output power increases, and the quasi-sinusoidal aspect of the modulation, which is seen at $1 \mathrm{~mW}$ [Fig. 6(a)], is distorted at $5 \mathrm{~mW}$ [Fig. 6(b)], especially when the crystal is inserted. This behavior is caused by the coupled Fabry-Perot effects that are due to the imperfect AR coating and to the gain-dependent semiconductor-laser phase-shift phenomenon. ${ }^{4}$ This could create wavelength zones with multiple thresholds to permit bistable operation. When the crystal is inserted, because it induces additional loss the relative value of the residual reflectivity of the AR coating with respect to the Littman-mounted grating becomes larger, which enhances the phenomenon. Nevertheless, because the quality of the AR coating, is sufficiently good, no bistability was observed, even at the maximum reachable power.

\section{Conclusions}

We have shown that the addition of a photorefractive crystal inside the cavity of a grating-tuned extendedcavity semiconductor laser prevents mode hopping and multimode operation by enhancing modal selectivity. The stabilizing filter dynamically adjusts itself to the lasing mode and operates in the whole tuning range of the ECL. Furthermore, we estimate that, for $1 \mathrm{~mW}$ of output power, the photorefractive grating builds up within $2 \mathrm{~ms}$. The continuoustuning speed that can be reached is thus estimated at $10 \mathrm{~nm} / \mathrm{s}$.

The authors acknowledge fruitful discussion with Jean-Luc Ayral of the Photonics Division, NetTest, Les Clayes sous Bois, France.

\section{References}

1. R. Ludeke and E. P. Harris, "Tunable GaAs laser in an external dispersive cavity," Appl. Phys. Lett. 20, 499-500 (1972).

2. R. Wyatt and W. J. Devlin, " $10 \mathrm{kHz}$ linewidth $1.5 \mu \mathrm{m}$ InGaAsP external cavity laser with $55 \mathrm{~nm}$ tuning range," Electron. Lett. 19, 110-112 (1983).

3. F. Favre and D. Le Guen, " $82 \mathrm{~nm}$ of continuous tunability for an external cavity semiconductor laser," Electron. Lett. 27, 183-184 (1991).

4. P. Zorabedian, "Axial-mode instability in tunable externalcavity semiconductor lasers," IEEE J. Quantum Electron. 30, 1542-1552 (1994).

5. M. de Labachelerie and G. Passedat, "Mode-hop suppression of Littrow grating-tuned lasers,” Appl. Opt. 32, 269-274 (1993).
6. M. de Labachelerie, H. Sasada, and G. Passedat, "Mode-hop suppression of Littrow grating-tuned lasers: erratum," Appl. Opt. 33, 3817-3819 (1994).

7. A. V. Yarovitskiǐ and V. L. Velichanskiǔ, "Limits of continuous frequency tuning of injection lasers with selective external cavities," Quantum Electron. 25, 765-769 (1995).

8. A. Godard, G. Pauliat, G. Roosen, P. Graindorge, and P. Martin, "Side-mode gain in grating-tuned extended-cavity semiconductor lasers: investigation of stable single-mode operation conditions," IEEE J. Quantum Electron. 38, 390401 (2002).

9. A. Godard, G. Pauliat, G. Roosen, P. Graindorge, and P. Martin, "Relaxation of the alignment tolerances of a $1.55-\mu \mathrm{m}$ extended-cavity semiconductor laser by use of an intracavity photorefractive filter," Opt. Lett. 26, 1955-1957 (2001).

10. A. Godard, G. Pauliat, G. Roosen, P. Graindorge, and P. Martin, "Stabilization of a $1.55 \mu \mathrm{m}$ extended-cavity semiconductor laser by intracavity dynamic holography," Eur. Phys. J. Appl. Phys. 20, 191-196 (2002).

11. L. Solymar, D. J. Webb, and A. Grunnet-Jepsen, "The physics and applications of photorefractive materials," in Oxford Series in Optical and Imaging Sciences, A. Hasegawa, M. Lapp, B. B. Snavely, H. Stark, A. C. Tam, and T. Wilson, eds. (Clarendon, Oxford, 1996), Vol. 11.

12. J. M. Ramsey and W. B. Whitten, "Controlled scanning of a continuous-wave dye laser with an intracavity photorefractive element," Opt. Lett. 12, 915-917 (1987).

13. N. Huot, J. M. Jonathan, G. Pauliat, P. Georges, A. Brun, and G. Roosen, "Laser mode manipulation by intracavity dynamic holography: application to mode selection,” Appl. Phys. B 69, 155-157 (1999).

14. S. Maerten, N. Dubreuil, G. Pauliat, G. Roosen, D. Rytz, and T. Salva, "Laser diode made single-mode by a self-adaptive photorefractive filter," Opt. Commun. 208, 183-189 (2002).

15. P. Delaye, L. A. de Montmorillon, I. Biaggio, J. C. Launay, and G. Roosen, "Wavelength dependent effective trap density in CdTe: evidence of the presence of two photorefractive species," Opt. Commun. 134, 580-590 (1997).

16. A. P. Bogatov, P. G. Eliseev, O. G. Okhotnikov, M. P. Rakhval'skiǔ, and K. A. Khaǐretdinov, "Interaction of modes and self-stabilization of single-frequency emission from injection lasers," Sov. J. Quantum Electron. 13, 1221-1229 (1983).

17. M. Yamada, "Theoretical analysis of nonlinear optical phenomena taking into account the beating vibration of the electron density in semiconductor lasers," J. Appl. Phys. 66, 81-89 (1989).

18. A. Uskov, J. Mørk, and J. Mark, "Wave mixing in semiconductor laser amplifiers due to carrier heating and spectral-hole burning," IEEE J. Quantum Electron. 30, 1769-1781 (1994). 